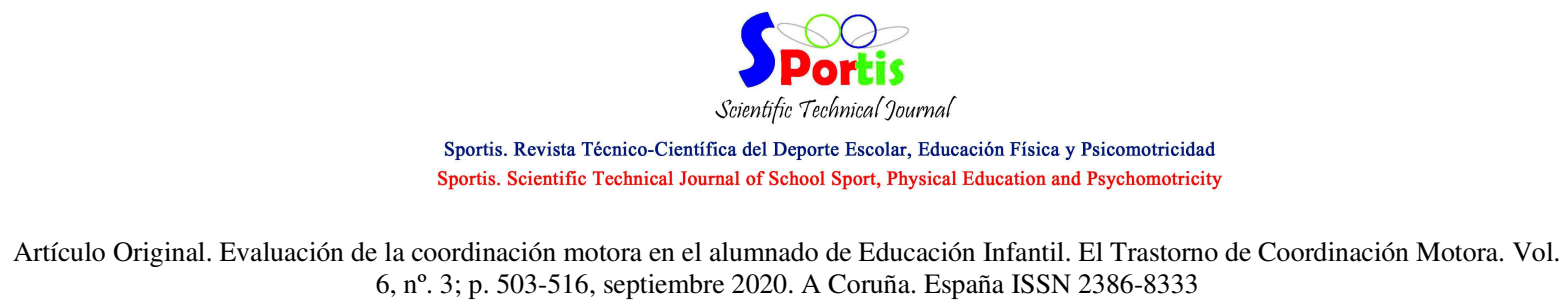

$6, n^{\circ} .3$; p. 503-516, septiembre 2020. A Coruña. España ISSN 2386-8333

\title{
Evaluación de la coordinación motora en alumnado de Educación Infantil. El Trastorno de Coordinación Motora
}

\section{Assessment of motor coordination in students of Early Childhood Education. Developmental Coordination Disorder}

\author{
Marta Parrado Merino; Adriana Nielsen Rodríguez; Ramón Romance García \\ Departamento de Didáctica de las Lenguas, las Artes y el Deporte. Laboratorio de \\ Motricidad Humana. Universidad de Málaga \\ Contacto: arromance@uma.es
}

Cronograma editorial: Artículo recibido: 08/04/2020 Aceptado:13/07/2020 Publicado: 01/09/2020

DOI: https://doi.org/10.17979/sportis.2020.6.3.6265

Fuente de financiación: Este trabajo ha sido financiado por la Universidad de Málaga (Campus de Excelencia Internacional Andalucía Tech) en el marco del Proyecto de Innovación Docente PIE19-111 y por el Ministerio de Educación, Cultura y Deporte a través de las ayudas para la Formación de Profesorado Universitario (FPU17/01554).

\section{Resumen}

El escaso desarrollo y adquisición de habilidades de coordinación, conocido como Trastorno de Coordinación Motora (TDC) o dispraxia del desarrollo, es un trastorno motor no atribuible a ningún tipo de discapacidad o trastorno neurológico de alteración del movimiento, pero que afecta a funciones tales como el habla, el lenguaje, la escritura o la atención. Se estima que su incidencia es del 5-15\% en niños en edad escolar, siendo la prevalencia mayor en varones. En el estudio participaron 91 alumnos de 5 años $(\overline{\mathrm{X}}=5,83 ; \mathrm{SD}=0,33)$ de la provincia de Málaga. Se evaluaron los niveles de adquisición de habilidades de coordinación motora, identificando los niños en situación de normalidad, en riesgo de disminución psicomotora o con sospecha de padecer TDC, y se analizaron las diferencias de desempeño motor en función del sexo. Para la valoración de la coordinación motora se recurrió a la versión española de la batería de evaluación del movimiento para niños (Movement Assessment Battery for Children) MABC2. Los resultados identifican a un 6,56\% de los niños como TDC y un 4,67\% en situación de riesgo. En cuanto a las diferencias asociadas al sexo, las niñas alcanzaron puntuaciones significativamente más altas en las áreas de destreza manual y de equilibrio, mientras que los

Para citar este artículo utilice la siguiente referencia: Parrado Merino, M.; Nielsen Rodríguez, A.; Romance García, R. (2020). Evaluación de la coordinación motora en alumnado de Educación Infantil. El Trastorno de Coordinación Motora. Sportis Sci J, 6 (3), 503-516.

DOI: https://doi.org/10.17979/sportis.2020.6.3.6265

http://revistas.udc.es/ 


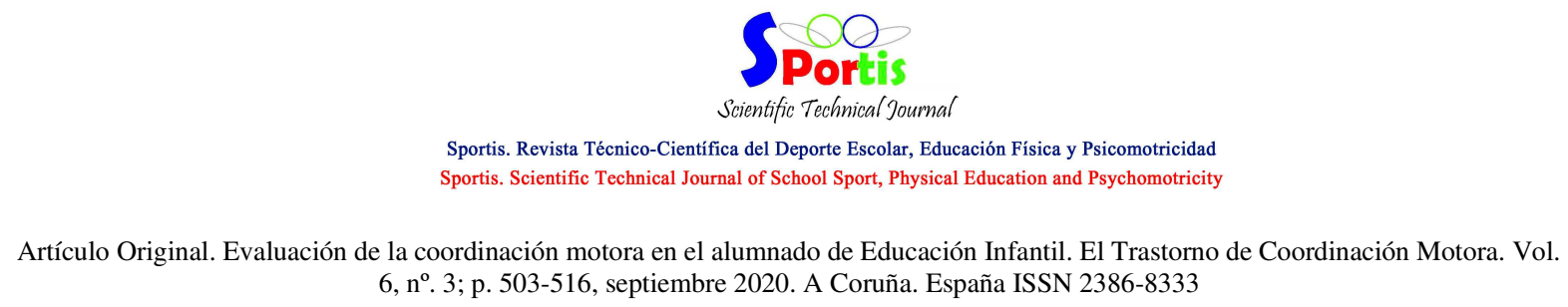

niños logran puntuaciones mayores, aunque no de manera significativa, en el área puntería y atrape.

Palabras clave: trastorno de Coordinación Motora; TDC; MABC; sexo; niños

\begin{abstract}
The lacking in coordination skills development and acquisition, known as Developmental Coordination Disorder (DCD) or developmental dyspraxia, is a motor disorder not attributable to any type of disability or neurological disorder of movement alteration, but which affects functions such as speech, language, writing or attention. Its influence is estimated to be $5-15 \%$ in school-age children, with the highest prevalence in boys. A total of 91 5-year-old students $(\bar{X}=5.83 ; S D=0.33)$ from the province of Malaga participated in the study. Acquisition levels of motor coordination skills were evaluated, identifying children in a normal situation, at risk of psychomotor decline or with suspected DCD, and the differences in motor performance according to sex were analyzed. For the assessment of motor coordination, the Spanish version of the Movement Assessment Battery for Children - Second Edition (MABC-2) was used. The results identify $6.56 \%$ of children as DCD and $4.67 \%$ at risk. Regarding the differences associated with sex, girls achieved significantly higher scores in manual dexterity and balance areas, while boys achieved higher scores, although not significantly, in aiming and catching area.
\end{abstract}

Keywords: developmental coordination disorder; DCD; MABC; sex; children

\title{
Introducción
}

Los primeros años de vida de una persona son clave en su desarrollo cognitivo y motor, pues durante la infancia se gestionará la constitución de su sistema nervioso. Crecimiento y actuarán paralelamente tratando de alcanzar niveles óptimos de desarrollo. Los niños y niñas alcanzan habilidades motoras básicas que les ayudarán a ir adquiriendo otras más complejas (Piek, Hands, \& Licari, 2012). Estas destrezas no solo son relevantes en la realización de ejercicio físico de una persona, sino que contribuyen a la mayoría de sus actividades diarias.

La manera de moverse por el entorno proporciona a la infancia nuevas oportunidades para aprender, repercutiendo también en su desarrollo cognitivo y social. Esta repercusión es bidireccional, pues los cambios cognitivos y sociales que experimenten influirán a su vez en la manera de relacionarse con el medio (Houwen, van der Veer, Visser, \& Cantell, 2017).

Para citar este artículo utilice la siguiente referencia: Parrado Merino, M.; Nielsen Rodríguez, A.; Romance García, R. (2020). Evaluación de la coordinación motora en alumnado de Educación Infantil. El Trastorno de Coordinación Motora. Sportis Sci J, 6 (3), 503-516.

DOI: https://doi.org/10.17979/sportis.2020.6.3.6265

http://revistas.udc.es/ 


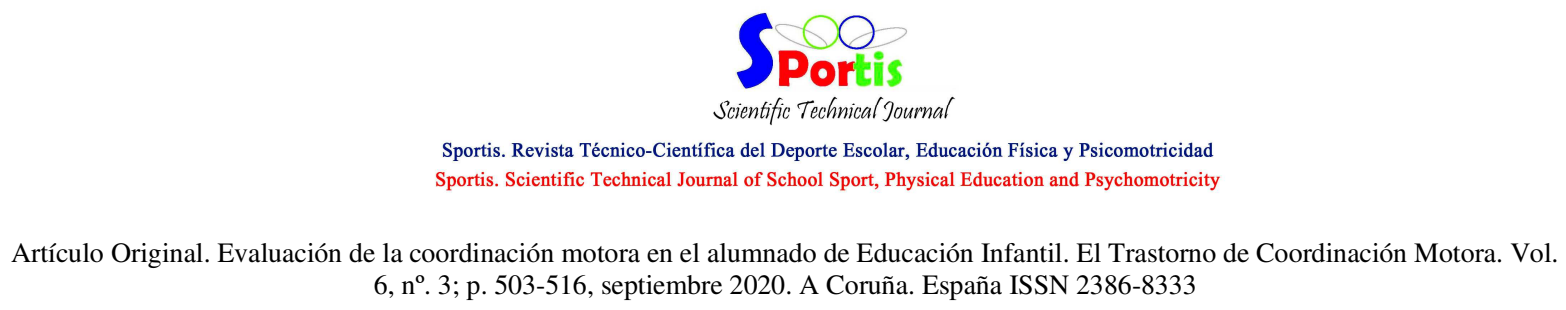

La trascendencia de la actividad física no ha pasado desapercibida, pudiendo asegurar que la práctica de esta ayuda a las niñas y niños a alcanzar un mejor estado de salud y desarrollo integral (Delgado \& Montes, 2017). Por ello, organizaciones como la Organización Mundial de la Salud se ha encargado de establecer unas recomendaciones mínimas de movimiento. Según dicha organización, un niño de entre 5 y 17 años deberían realizar al menos 60 minutos al día de actividad física moderada-vigorosa, incluyendo en ellas actuaciones específicas para fortalecer músculos y huesos al menos tres veces a la semana (OMS, 2010). Aunque estas recomendaciones no contemplan edades inferiores a los 5 años, debemos tener en cuenta la relevancia del movimiento como factor estimulador del desarrollo de las capacidades físicas y cognitivas (National Association for Sport and Physical Education, 2012; UNESCO, 2015).

En un alto porcentaje de casos, estas recomendaciones no son atendidas debido al estilo de vida sedentario que la sociedad ha adoptado de forma general (OMS, 2010). A este hecho debemos sumar que, en la mayor parte de los colegios, las horas dedicadas al trabajo de psicomotricidad en Educación Infantil son escasas.

El sedentarismo infantil de hoy día puede conducir a un pobre o deficitario desarrollo psicomotor, dando lugar a disfunciones y afectando a diversas áreas de desarrollo sin que realmente exista ninguna patología causante. Este trastorno, que cada vez es más frecuente, se conoce como Trastorno de Coordinación Motora (TDC) o dispraxia del desarrollo.

El Trastorno de Coordinación Motora es un trastorno motor del neurodesarrollo contemplado en el Manual diagnóstico y estadístico de los trastornos mentales-V (DSM-5) y en la Clasificación internacional de enfermedades-10 (CIE-10) (Asociación Americana de Psiquiatría, 2014), y viene caracterizado por un escaso desarrollo y adquisición de habilidades de coordinación, lo que afecta además a otras habilidades o funciones tales como el habla, al lenguaje, la escritura o la atención (Henderson, 2012).

La presencia de Trastorno de Coordinación Motora no puede ser atribuible a discapacidad intelectual o visual, ni a trastornos neurológicos que alteran al movimiento (Blank, Smits-Engelsman, Polatajko, \& Wilson, 2012). Estos problemas en el desarrollo motor tienen consecuencias negativas en los procesos de aprendizaje (Solís, Prieto, Nistal, \&

Para citar este artículo utilice la siguiente referencia: Parrado Merino, M.; Nielsen Rodríguez, A.; Romance García, R. (2020). Evaluación de la coordinación motora en alumnado de Educación Infantil. El Trastorno de Coordinación Motora. Sportis Sci J, 6 (3), 503-516.

DOI: https://doi.org/10.17979/sportis.2020.6.3.6265

http://revistas.udc.es/ 


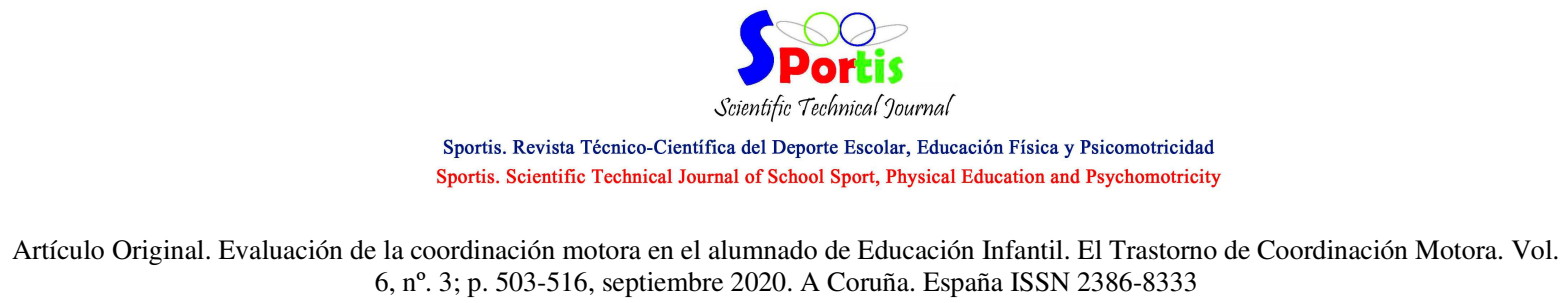

Vázquez, 2017), por lo que las niñas y niños que presentan Trastorno de Coordinación Motora tienen más probabilidades de no transitar bien la escuela (Magalhães, Cardoso, \& Missiuna, 2011) y de sufrir problemas de relación social, generándose en ellos además sentimientos y emociones negativas.

Sin una intervención apropiada ante casos de Trastorno de Coordinación Motora, las dificultades de movimiento y de coordinación persistirán en el tiempo, creando además otros problemas y provocando que el niño alcance la edad adulta sin desprenderse de los mismos (Henderson, 2012).

Se estima que el Trastorno de Coordinación Motora afecta, aproximadamente, al 5$15 \%$ de los niños en edad escolar, siendo su prevalencia mayor en varones (Blank et al., 2012) (Lingam, Hunt, Golding, Jongmans, \& Emond, 2009). A pesar de su frecuencia y trascendencia, el Trastorno de Coordinación Motora es aún desconocido para una gran parte de los profesionales sociosanitarios y educativos (Blank et al., 2012; Missiuna, Moll, King, King, \& Law, 2007; Missiuna, Moll, Law, King, \& King, 2006) , y al día de hoy ha sido poco estudiada en población española (Plata \& Garbiñe, 2009).

Aunque el Trastorno de Coordinación Motora no puede ser atribuible a discapacidad intelectual o visual, ni a trastornos neurológicos que alteran al movimiento sí que tiene gran comorbilidad con otras alteraciones o trastornos del neurodesarrollo, por ejemplo, con el Trastorno por Déficit de Atención con Hiperactividad o TDAH (Blank et al., 2012).

Visto lo anterior, definimos como objetivos de este trabajo (I) estimar los niveles de adquisición de habilidades de coordinación motora en los niños y niñas de 5 años, (II) analizar si existen diferencias significativas en el desempeño motor asociadas al sexo y (III) discernir el número de casos de niños y niñas en situación de normalidad, o de riesgo de disminución psicomotora, o con sospecha de Trastorno de Coordinación Motora.

\section{Material y método}

Para la evaluación del nivel de coordinación motora de los niños y niñas se empleó la versión española de la Batería de Evaluación del Movimiento para Niños - Segunda edición (Movement Assessment Battery for Children - Second Edition, MABC-2) (Henderson, 2012).

\footnotetext{
Para citar este artículo utilice la siguiente referencia: Parrado Merino, M.; Nielsen Rodríguez, A.; Romance García, R. (2020). Evaluación de la coordinación motora en alumnado de Educación Infantil. El Trastorno de Coordinación Motora. Sportis Sci J, 6 (3), 503-516. DOI: https://doi.org/10.17979/sportis.2020.6.3.6265

http://revistas.udc.es/ 
Esta batería fue creada por Henderson y Sudgen, en 1992, aunque ha sufrido algunas modificaciones desde entonces. Hoy día es una de las más empleadas para identificar a niños y niñas con problemas de desarrollo motor (Ayán, 2017).

La batería MABC-2 se basa en la intervención ecológica, que defiende la inseparable conexión entre las áreas motora, cognitiva y emocional de una persona (Henderson, 2012).

El test estandarizado tiene como objetivo identificar y describir la capacidad motriz del niño o la niña en cuestión. Consiste en la realización de ocho pruebas concretas de una manera rigurosamente establecida. Las pruebas a realizar se dividen en tres dimensiones: destreza manual, puntería y atrape, y equilibrio. Algunos otros aspectos que mide el test son la motricidad fina, la velocidad, la agilidad y la fuerza.

La Batería MABC-2 (Henderson, 2012) permite la evaluación y detección del Trastorno de Coordinación Motora en los niños y niñas de entre 4 y 16 años. Los estudios en los que ha sido empleada son de diversa índole; por ejemplo Houwen et al. (2017) evaluaron el rendimiento motor de niños y niñas de entre 3 y 5 años para tratar de relacionar los resultados obtenidos con las funciones ejecutivas de los participantes. Este estudio analizó las diferencias de desempaño teniendo en cuenta la edad, el sexo, el estatus socioeconómico y la presencia de Trastorno por Déficit de Atención con Hiperactividad. Otros estudios emplearon la Batería MABC-2 para realizar comparaciones entre grupos: niños que tienen un trasplante y niños que no lo tienen (Almaas et al., 2015); evaluar el nivel de coordinación antes y después de un entrenamiento o evaluar y comparar la población infantil (Delgado-Lobete \& Montes-Montes, 2017).

\title{
Participantes
}

En este estudio transversal de prevalencia participaron 91 alumnos de Educación Infantil, de los cuales 43 eran niñas ( 7 con predominancia lateral izquierda) y 48 niños (10 con predominancia lateral izquierda) con una media de edad de 5 años y 10 meses y una desviación típica de 4 meses $(\overline{\mathrm{X}}=5,83 ; \mathrm{SD}=0,33)$, procedentes de 5 centros educativos de la provincia de Málaga.

Para citar este artículo utilice la siguiente referencia: Parrado Merino, M.; Nielsen Rodríguez, A.; Romance García, R. (2020). Evaluación de la coordinación motora en alumnado de Educación Infantil. El Trastorno de Coordinación Motora. Sportis Sci J, 6 (3), 503-516.

DOI: https://doi.org/10.17979/sportis.2020.6.3.6265

http://revistas.udc.es/ 


\section{Análisis estadístico}

Para el tratamiento de los datos se empleó el paquete estadístico IBM SPSS $20^{\circledR}$ en su versión para Windows tras ser estos previamente preparados con Microsoft Excel ${ }^{\circledR}$. Los datos se expresaron a través de porcentajes, medias y desviaciones estándar, mientras que para determinar la diferencia de medias entre dos variables independientes cualitativas se utilizó la prueba U de Mann-Whitney.

\section{Resultados}

Tras la aplicación de la Batería MABC-2 y habiendo procedido al análisis de los datos obtenidos, todo ello de acuerdo con las indicaciones y especificaciones proporcionadas en el manual de la prueba, encontramos que los resultados (expresados en percentiles) más elevados se obtienen, de forma general, en el área de Equilibrio $(\overline{\mathrm{X}}=32,35)$, seguidos por el área de Destreza Manual $(\overline{\mathrm{X}}=25,07)$ y, por último, el área de Puntería y Atrape $(\overline{\mathrm{X}}=17,07)$, siendo la media global obtenida al considerar todas las áreas sometidas al test mediante la batería de $\bar{X}=38,20$ (Tabla $\left.{ }^{\circ} 1\right)$.

Tabla $\mathrm{n}^{\circ} 1$. Medias y Desviaciones Típicas de las variables analizadas en el estudio.

\begin{tabular}{lcccccc}
\hline & $\overline{\mathrm{X}}$ & $\mathrm{SD}$ & $\overline{\mathrm{x}}$ Niñas & $\begin{array}{c}\text { SD } \\
\text { Niñas }\end{array}$ & $\begin{array}{c}\overline{\mathrm{x}} \\
\text { Niños }\end{array}$ & $\begin{array}{c}\text { SD } \\
\text { Niños }\end{array}$ \\
\hline MABC-2 Percentil & & & & & & \\
Destreza Manual & 25,07 & 6,56 & 40,3605 & 32,71146 & 24,2104 & 22,88760 \\
Puntería y Atrape & 17,07 & 4,67 & 29,1628 & 20,48423 & 38,2833 & 28,73052 \\
Equilibrio & 32,35 & 3,68 & 70,9767 & 20,63110 & 60,2917 & 24,46966 \\
Puntuación Total & 38,20 & 25,44 & 43,5581 & 25,88648 & 33,3958 & 24,29669 \\
\hline
\end{tabular}

$\overline{\text { MABC-2 Percentil = Resultado en percentil obtenido en la Batería de evaluación del movimiento para niños. }}$

En cuanto a las diferencias asociadas al sexo (Tabla $n^{\circ} 1$, Figura ${ }^{\circ} 1$, Figura $n^{\circ} 2$, Figura $\mathrm{n}^{\circ} 3$ y Figura $\mathrm{n}^{\circ} 4$ ), se observa una tendencia de las niñas a alcanzar puntuaciones significativamente mayores que los niños en las áreas de Equilibrio ( $\overline{\mathrm{X}}$ niñas=70,9767; $\overline{\mathrm{X}}$ niños=60,2917) (Figura n³) y de Destreza Manual ( $\bar{x}$ niñas=40,3605; $\bar{x}$ niños=24,2104) 
Artículo Original. Evaluación de la coordinación motora en el alumnado de Educación Infantil. El Trastorno de Coordinación Motora. Vol. $6, n^{\circ} .3$; p. 503-516, septiembre 2020. A Coruña. España ISSN 2386-8333

(Figura $\mathrm{n}^{\circ} 1$ ), mientras que los niños obtienen puntuaciones mayores en el área de Puntería y Atrape $(\bar{X}$ niñas $=29,1628 ; \bar{X}$ niños $=38,2833)$ (Figura $\left.n^{\circ} 2\right)$, si bien en este caso la diferencia no es significativa (Tabla $\left.\mathrm{n}^{\circ} 2\right)$.

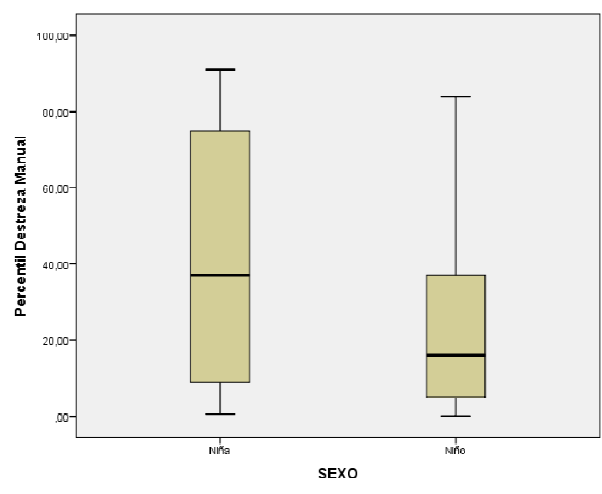

Figura $\mathrm{n}^{\circ} 1$. Distribución de los resultados de Destreza Manual expresados en percentiles.

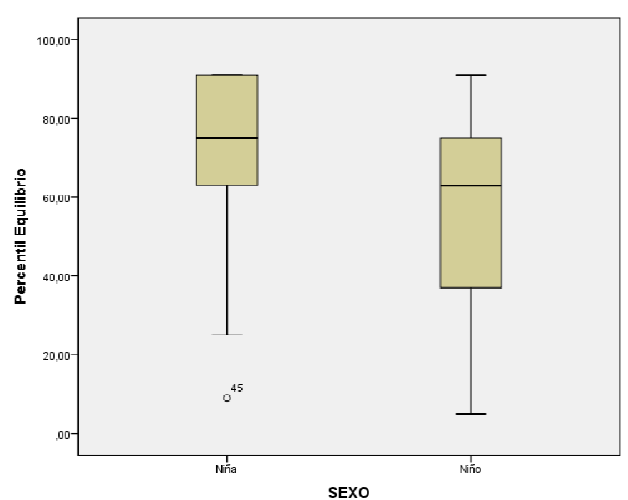

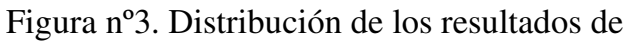
destreza de Equilibrio expresado en percentiles.

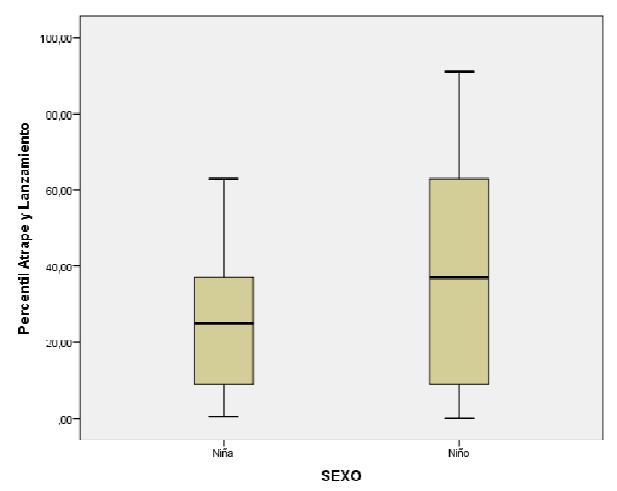

Figura $n^{\circ} 2$. Distribución de los resultados de la destreza de Puntería y Atrape expresado en percentiles.

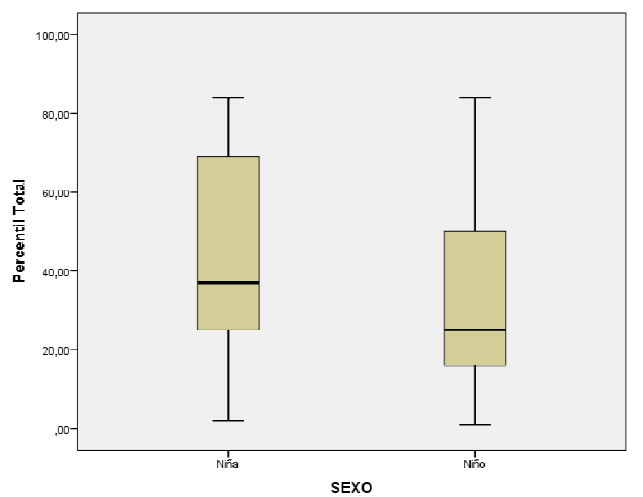

Figura $\mathrm{n}^{\circ} 4$. Distribución de los resultados totales expresado en percentiles.

Tabla $n^{\circ} 2$. Estadístico de contraste para grupo de niños y niña.

\begin{tabular}{ccccc}
\hline & $\begin{array}{c}\text { Destreza } \\
\text { Manual }\end{array}$ & $\begin{array}{c}\text { Lanzamiento } \\
\text { y atrape }\end{array}$ & Equilibrio & $\begin{array}{c}\text { Puntuación } \\
\text { Total }\end{array}$ \\
\hline U de Mann- & 763,500 & 867,000 & 755,500 & 797,000 \\
$\begin{array}{c}\text { Whitney } \\
\text { Sig. asintótica } \\
\text { (bilateral) }\end{array}$ & $.032^{*}$ & .185 & $.026^{*}$ & .060 \\
\hline
\end{tabular}

Nota: $* \mathrm{p}<.05 ; * * \mathrm{p}<.01$

Para citar este artículo utilice la siguiente referencia: Parrado Merino, M.; Nielsen Rodríguez, A.; Romance García, R. (2020). Evaluación de la coordinación motora en alumnado de Educación Infantil. El Trastorno de Coordinación Motora. Sportis Sci J, 6 (3), 503-516.

DOI: https://doi.org/10.17979/sportis.2020.6.3.6265 
Finalmente, los resultados muestran que un $9,89 \%$ de los niños y niñas evaluados padecen Trastorno de Coordinación Motora lo cual y que el 7,69\% se encuentra en situación de riesgo de padecerlo, lo cual supone un porcentaje total del 17,58\% de niños y niñas en situación de sospecha más o menos firme de padecer Trastorno de Coordinación Motora (Tabla n³ y Figura nº5).

Tabla $\mathrm{n}^{\circ} 3$. Frecuencia y porcentaje de los resultados obtenidos en el MABC-2.

\begin{tabular}{lcc}
\hline & Frecuencia & $\%$ \\
\hline TDC & 9 & 9,89 \\
En riesgo & 7 & 7,69 \\
Normal & 75 & 82,42
\end{tabular}

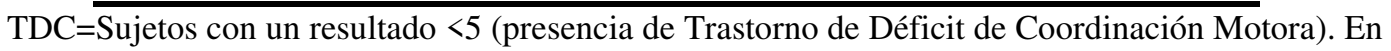
riesgo $=$ Sujetos con sospecha de padecer TDC. Normal=Sujetos con resultados óptimos.

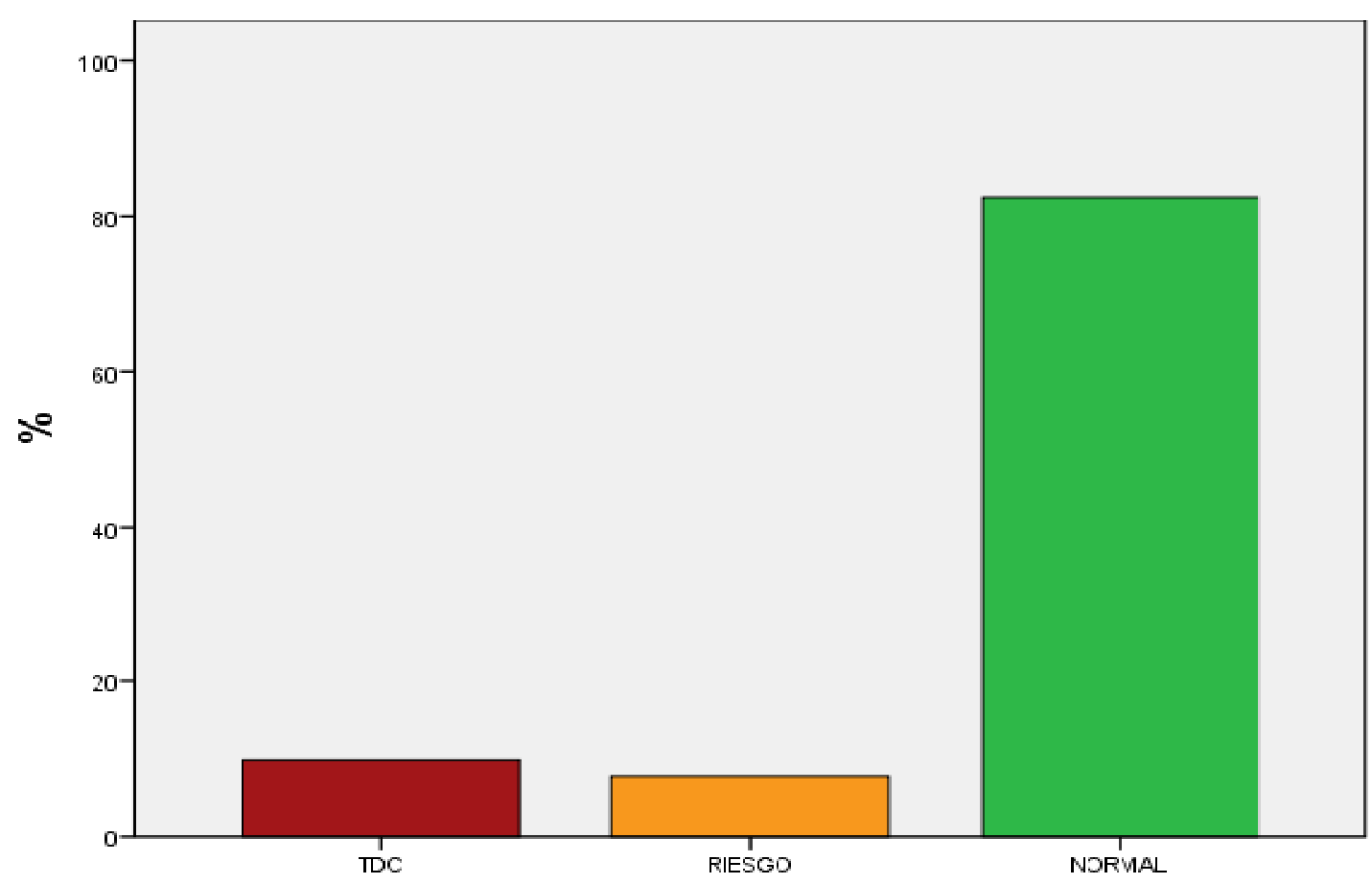

Figura ${ }^{\circ} 5$. Distribución en porcentajes de los casos considerados como TDC $=$ Sujetos con un resultado $<5$ (presencia de Trastorno de Déficit de Coordinación Motora). En riesgo=Sujetos con sospecha de padecer TDC. Normal=Sujetos con resultados óptimos. 


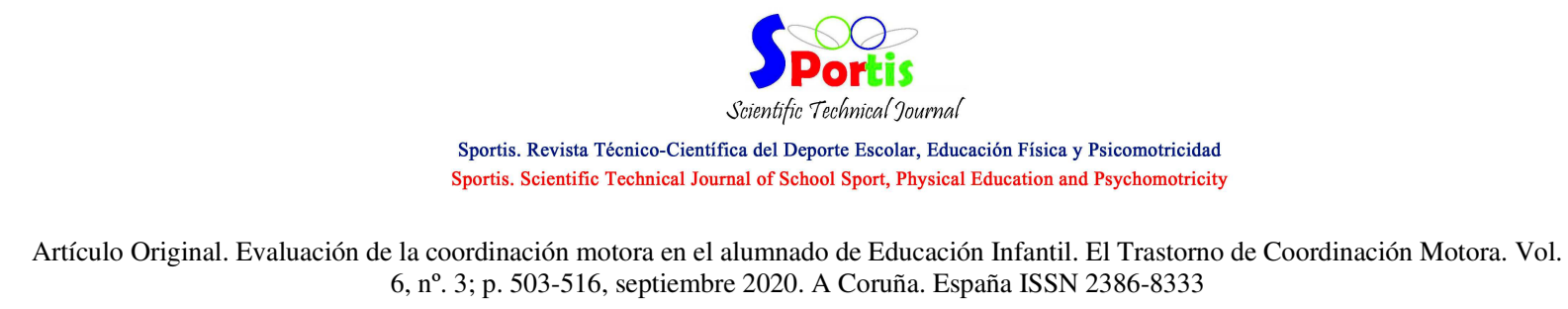

\section{Discusión}

El propósito de este estudio fue estimar los niveles de adquisición de habilidades de coordinación motora en los niños y niñas de 5 años, al mismo tiempo que se ha comprobado si existen diferencias significativas en el desempeño motor asociadas al sexo y se ha establecido el número de casos de niños y niñas en situación de normalidad, en riesgo de disminución psicomotora o con sospecha de Trastorno de Coordinación Motora.

Como resultado, se ha revelado información trascendente sobre el uso de la Batería MABC-2 en una muestra poblacional de niños y niñas de Educación Infantil con una media de edad de 5 años y 10 meses y una desviación típica de 4 meses ( $\bar{X}=5,83 ; \mathrm{SD}=0,33)$.

Como podemos apreciar en los datos recogidos en la Tabla $n^{\circ} 1$, la muestra de estudio de esta investigación se encuentra en el percentil 25,07 en el área de Destreza Manual, mientras que en el área de Puntería y Atrape se encuentra en el percentil 17,07 y en el área de Equilibrio en 32,35. En total, la media obtenida al sumar los resultados de todas las áreas que se someten a evaluación con la batería es de 38,20, lo cual se aproxima al resultado de otras investigaciones similares (Delgado-Lobete \& Montes-Montes, 2017), así como al resultado general que sugiere la propia Batería MABC-2 como normal en su manual.

En cuanto a las diferencias en función del sexo (Tabla $n^{\circ} 1$ ), tanto en las actividades del área de Destreza Manual como en las actividades del área de Equilibrio, las chicas tienden consistentemente a alcanzar puntuaciones más altas que los chicos de forma significativa (Tabla $n^{\circ} 2$, Figura $n^{\circ} 1$ y Figura $n^{\circ} 3$ ). Sin embargo, los niños logran puntuaciones mayores, aunque no de manera significativa, en el área Puntería y Atrape (Figura n²). Estos resultados son análogos a los de estudios anteriores en los que las niñas demuestran mejores habilidades de motricidad fina, mientras que los niños obtienen mayor puntuación en actividades de lanzar objetos (Flatters, Hill, Williams, Barber, \& Mon-Williams, 2014). En nuestro caso, podemos confirmar un desarrollo de la coordinación motora global superior en las niñas que en los niños de esta edad (Figura $n^{\circ} 4$ ), aunque no de forma significativa. Estos resultados están en concordancia con los obtenidos por otros estudios llevados a cabo en la población escolar (Rivard, Missiuna, McCauley, \& Cairney, 2014), en los que se sugiere que tal vez el

Para citar este artículo utilice la siguiente referencia: Parrado Merino, M.; Nielsen Rodríguez, A.; Romance García, R. (2020). Evaluación de la coordinación motora en alumnado de Educación Infantil. El Trastorno de Coordinación Motora. Sportis Sci J, 6 (3), 503-516.

DOI: https://doi.org/10.17979/sportis.2020.6.3.6265

http://revistas.udc.es/ 


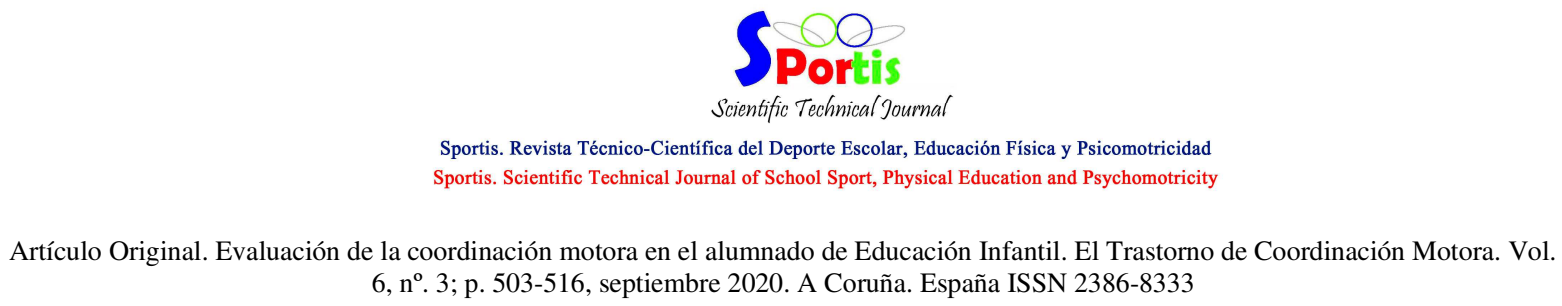

mayor grado de desarrollo que suelen mostrar las chicas haga más significativa esta diferencia en cuanto al sexo en relación al nivel de coordinación alcanzado.

Por otro lado, se puede apreciar cómo el porcentaje de niños identificados como con alta posibilidad de padecer Trastorno de Coordinación Motora es del 9,89\%, lo cual sumado al 7,69\% que se encuentra en situación de riesgo de riesgo de padecerlo, supone un porcentaje total de $17,58 \%$ de los niños y niñas en situación de sospecha de padecer Trastorno de Coordinación Motora (Tabla $n^{\circ} 3$ y Figura $n^{\circ} 5$ ). Estas cifras se corresponden, aunque son ligeramente superiores, a los resultados obtenidos en otros estudios a nivel internacional, que sitúan la prevalencia de este trastorno en torno al 5-15\% de los niños en edad escolar (Blank et al., 2012; Lingam et al., 2009), aunque puede superar el $24 \%$ atendiendo a los instrumentos utilizados para su identificación (Valentini, Oliveira, Pangelinan, Whitall, \& Clark, 2017).

A pesar de su relevancia para el correcto desarrollo infantil, el Trastorno de Coordinación Motora es un trastorno bastante desconocido en España (Leonard \& Hill, 2015; Wenzel, 2017) y apenas se han realizado investigaciones al respecto (Plata \& Garbiñe, 2009). Sin embargo, conocer la prevalencia de Trastorno de Coordinación Motora en niños en la edad infantil es esencial debido a la influencia que tiene sobre la vida social y afectiva de los niños, así como en el rendimiento escolar y académico. Un diagnóstico temprano de este problema permitiría un rápido tratamiento y una intervención con mayores posibilidades de éxito. Algunos autores han informado que los niños con Trastorno de Coordinación Motora presentan dificultades significativas en la lectoescritura, las matemáticas, la atención y las habilidades sociales y comunicativas (Harrowell, Hollén, Lingam, \& Emond, 2018). Otros autores (Bo et al., 2014; Huau, Velay, \& Jover, 2015; Prunty, Barnett, Wilmut, \& Plumb, 2016) afirmaron que estos niños también tienen alteraciones en las habilidades grafomotoras y en la escritura, especialmente en la precisión y velocidad de ejecución y en la consistencia temporal posiblemente asociadas a dificultades en la percepción visual (Prunty et al., 2016). La comprensión, la lógica matemática y concretamente el procesamiento numérico simbólico y no simbólico (Gomez et al., 2015) también se ven afectados por este trastorno, lo que se ha relacionado con una menor destreza en el seguimiento visual (Gomez, Piazza, Jobert, Dehaene-Lambertz, \& Huron, 2017).

Para citar este artículo utilice la siguiente referencia: Parrado Merino, M.; Nielsen Rodríguez, A.; Romance García, R. (2020). Evaluación de la coordinación motora en alumnado de Educación Infantil. El Trastorno de Coordinación Motora. Sportis Sci J, 6 (3), 503-516. DOI: https://doi.org/10.17979/sportis.2020.6.3.6265

http://revistas.udc.es/ 


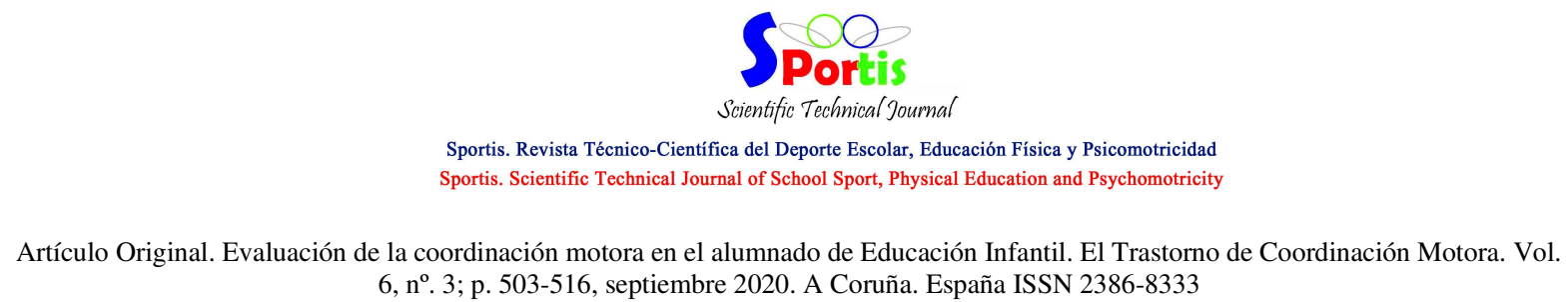

$6, n^{\circ} .3$; p. 503-516, septiembre 2020. A Coruña. España ISSN 2386-8333

\section{Conclusiones}

El Trastorno de Coordinación Motora es un trastorno que, a pesar de ser bastante desconocido, afecta la vida social, a la vida afectiva y al rendimiento escolar y académico de los niños. Es este un problema que no puede ser atribuible a ningún tipo de discapacidad intelectual o visual, ni a trastornos neurológicos que alteren al movimiento. Sin embargo, sí que tiene gran comorbilidad con otras alteraciones o trastornos del neurodesarrollo.

En este trabajo hemos evaluado distintas variables relacionadas con los niveles de adquisición de habilidades de coordinación motora en niños y niñas de 5 años, obteniendo unos resultados que muestran que el porcentaje de niños que presentan o están en riesgo de presentar Trastorno de Coordinación Motora es del 17,58\% (9,89\% con alta probabilidad y $7,69 \%$ en situación de riesgo), lo cual sobrepasa levemente los parámetros establecidos y no deja de ser preocupante debido a las consecuencias que puede acarrear para este tanto por ciento relativamente alto de la población infantil.

Además, nuestros resultados muestran de una forma significativa que las niñas de 5 años poseen un mayor nivel general de coordinación en las habilidades motoras en general, y en las habilidades manuales y de equilibrio más concretamente. Por otro lado, los niños presentan un mayor nivel de coordinación motora en las habilidades relacionadas con el lanzamiento y el atrape, aunque esta diferencia no llega a ser significativa como ocurre en el caso anterior.

Debido a la importante influencia que el Trastorno de Coordinación Motora tiene sobre el desarrollo social, intelectual, afectivo y motor de los niños, así como el desconocimiento que aún existe sobre este trastorno, se hace necesario incrementar el número de investigaciones que aborden esta problemática para acercar tanto a la comunidad científica como a la comunidad educativa no solo la información que les permita conocer este trastorno, sino las pautas de actuación en casos de niños que lo presenten y las posibles vías de actuación para prevenirlo desde las primeras edades.

\footnotetext{
Para citar este artículo utilice la siguiente referencia: Parrado Merino, M.; Nielsen Rodríguez, A.; Romance García, R. (2020). Evaluación de la coordinación motora en alumnado de Educación Infantil. El Trastorno de Coordinación Motora. Sportis Sci J, 6 (3), 503-516. DOI: https://doi.org/10.17979/sportis.2020.6.3.6265

http://revistas.udc.es/
} 


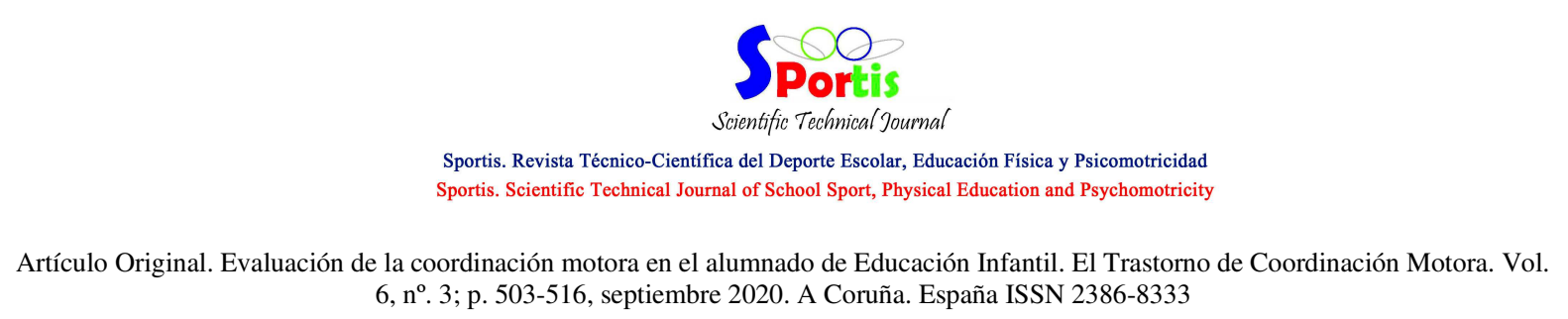

\section{Agradecimientos}

Este trabajo ha sido financiado por la Universidad de Málaga (Campus de Excelencia Internacional Andalucía Tech) en el marco del Proyecto de Innovación Docente PIE19-111 y por el Ministerio de Educación, Cultura y Deporte a través de las ayudas para la Formación de Profesorado Universitario (FPU17/01554).

\section{Referencias}

Almaas, R., Jensen, U., Loennecken, M. C., Tveter, A. T., Sanengen, T., Scholz, T., \& Holm, I. (2015). Impaired Motor Competence in Children With Transplanted Liver. Journal of Pediatric Gastroenterology and Nutrition, 60(6), 723-728. DOI: https://doi.org/10.1097/MPG.0000000000000757

Asociación Americana de Psicología (2014). Manual diagnóstico y estadístico de los trastornos mentales. Madrid: Panamericana.

Ayán, C. (2017). La valoración de la condición física en la educación infantil: Principales tests de aplicación: Paidotribo.

Blank, R., Smits-Engelsman, B., Polatajko, H., \& Wilson, P. (2012). European Academy for Childhood Disability (EACD): Recommendations on the definition, diagnosis and intervention of developmental coordination disorder (long version)*. Developmental Medicine \& Child Neurology, 54(1), 54-93. DOI: https://doi.org/10.1111/j.14698749.2011.04171.x

Bo, J., Colbert, A., Lee, C.-M., Schaffert, J., Oswald, K., \& Neill, R. (2014). Examining the relationship between motor assessments and handwriting consistency in children with and without probable developmental coordination disorder. Research in developmental disabilities, 35(9), 2035-2043. DOI: https://doi.org/10.1016/j.ridd.2014.04.027

Delgado-Lobete, L., \& Montes-Montes, R. (2017). Perfil y desarrollo psicomotor de los niños españoles entre 3 y 6 años. Sportis, 3(3), 454-470. DOI: https://doi.org/10.17979/sportis.2017.3.3.2002

Flatters, I., Hill, L. J. B., Williams, J. H. G., Barber, S. E., \& Mon-Williams, M. (2014). Manual control age and sex differences in 4 to 11 year old children. PloS one, 9(2), e88692. DOI: https://doi.org/10.1371/journal.pone.0088692

Gomez, A., Piazza, M., Jobert, A., Dehaene-Lambertz, G., Dehaene, S., \& Huron, C. (2015). Mathematical difficulties in developmental coordination disorder: Symbolic and nonsymbolic number processing. Research in Developmental Disabilities, 43, 167178. DOI: https://doi.org/10.1016/j.ridd.2015.06.011

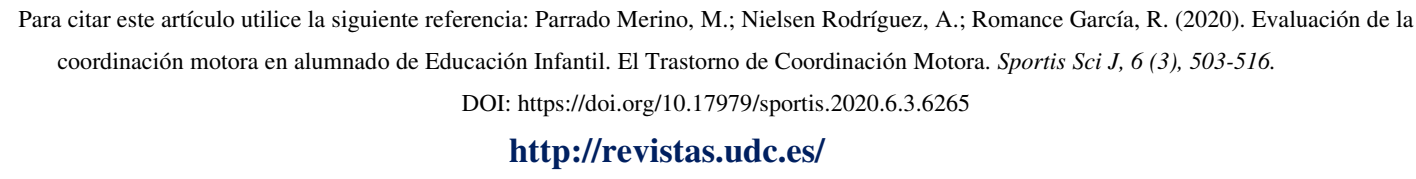


Gomez, A., Piazza, M., Jobert, A., Dehaene-Lambertz, G., \& Huron, C. (2017). Numerical abilities of school-age children with Developmental Coordination Disorder (DCD): A behavioral and eye-tracking study. Human movement science, 55, 315-326. DOI: https://doi.org/10.1016/j.humov.2016.08.008

Harrowell, I., Hollén, L., Lingam, R., \& Emond, A. (2018). The impact of developmental coordination disorder on educational achievement in secondary school. Research in developmental disabilities, 72, 13-22. DOI: https://doi.org/10.1016/j.ridd.2017.10.014

Henderson, S. E. (2012). Batería de evaluación del movimiento para niños-2 : (MABC-2). In D. A. Sugden, A. L. Barnett, L. M. Ruiz Pérez, \& J. L. Graupera Sanz (Eds.), MABC2. Madrid: Pearson.

Houwen, S., van der Veer, G., Visser, J., \& Cantell, M. (2017). The relationship between motor performance and parent-rated executive functioning in 3- to 5-year-old children: What is the role of confounding variables? Human Movement Science, 53, 24-36. DOI: https://doi.org/10.1016/j.humov.2016.12.009

Huau, A., Velay, J.-L., \& Jover, M. (2015). Graphomotor skills in children with developmental coordination disorder (DCD): Handwriting and learning a new letter. Human movement science, 42, 318-332. DOI: https://doi.org/10.1016/j.humov.2015.03.008

Leonard, H. C., \& Hill, E. L. (2015). Executive difficulties in developmental coordination disorder: methodological issues and future directions. Current Developmental Disorders Reports, 2(2), 141-149. DOI: https://doi.org/10.1007/s40474-015-0044-8

Lingam, R., Hunt, L., Golding, J., Jongmans, M., \& Emond, A. (2009). Prevalence of developmental coordination disorder using the DSM-IV at 7 years of age: A UK population-based study. American Academy of Pediatrics, 123, e693-e700. DOI: https://doi.org/10.1542/peds.2008-1770

Magalhães, L. C., Cardoso, A. A., \& Missiuna, C. (2011). Activities and participation in children with developmental coordination disorder: A systematic review. Research in Developmental Disabilities, 32(4), 1309-1316. DOI: https://doi.org/10.1016/j.ridd.2011.01.029

Missiuna, C., Moll, S., King, S., King, G., \& Law, M. (2007). A Trajectory of Troubles. Physical \& Occupational Therapy In Pediatrics, 27(1), 81-101. DOI: https://doi.org/10.1300/J006v27n01_06

Missiuna, C., Moll, S., Law, M., King, S., \& King, G. (2006). Mysteries and Mazes: Parents' Experiences of Children with Developmental Coordination Disorder. Canadian Journal of Occupational Therapy, 73(1), 7-17. DOI: https://doi.org/10.2182/cjot.05.0010 
National Association for Sport and Physical Education (2012). 2012 shape of the nation report: Status of physical education in the USA: American Alliance for Health.

Organización Mundial de la Salud (2010). Recomendaciones mundiales sobre actividad física para la salud. Suiza: Organización Mundial de la Salud.

Piek, J. P., Hands, B., \& Licari, M. K. (2012). Assessment of Motor Functioning in the Preschool Period. Neuropsychology Review, 22(4), 402-413. DOI: https://doi.org/10.1007/s11065-012-9211-4

Plata, R., \& Garbiñe, B. (2009). El niño con trastorno del desarrollo de la coordinación ¿Un desconocido en nuestra comunidad? Norte de Salud Mental, 8, 18-30.

Prunty, M., Barnett, A. L., Wilmut, K., \& Plumb, M. (2016). Visual perceptual and handwriting skills in children with Developmental Coordination Disorder. Human movement science, 49, 54-65. DOI: https://doi.org/10.1016/j.humov.2016.06.003

Rivard, L., Missiuna, C., McCauley, D., \& Cairney, J. (2014). Descriptive and factor analysis of the Developmental Coordination Disorder Questionnaire (DCDQ '07) in a population-based sample of children with and without Developmental Coordination Disorder. Child: care, health and development, 40(1), 42-49. DOI: https://doi.org/10.1111/j.1365-2214.2012.01425.x

UNESCO (2015). Educación Física de Calidad. Guía para los responsables políticos. Francia: UNESCO.

Valentini, N. C., Oliveira, M. A., Pangelinan, M. M., Whitall, J., \& Clark, J. E. (2017). Can the MABC discriminate and predict motor impairment? A comparison of Brazilian and American children. International Journal of Therapy and Rehabilitation, 24(3), 105-113. DOI: https://doi.org/10.12968/ijtr.2017.24.3.105

Wenzel, A. (2017). The sage encyclopedia of abnormal and clinical psychology (Vol. 1): SAGE Publications. DOI: https://doi.org/10.4135/9781483365817 